\title{
Post-Eruption Arcades and Interplanetary Coronal Mass Ejections
}

\author{
S. Yashiro ${ }^{1,2}$, N. Gopalswamy ${ }^{2}$, P. Mäkelä ${ }^{1,2}$, and S. Akiyama ${ }^{1,2}$ \\ 1: The Catholic University of America, Washington, DC 20064, USA \\ 2: NASA Goddard Space Flight Center, Greenbelt, MD 20771, USA \\ seiji.yashiro@nasa.gov; nat.gopalswamy@nasa.gov; pertti.makela@nasa.gov; \\ sachiko.akiyama@nasa.gov
}

\begin{abstract}
We compare the temporal and spatial properties of post-eruption arcades (PEAs) associated with coronal mass ejections (CMEs) at the Sun that end up as magnetic cloud (MC) and non-MC events in the solar wind. We investigate the length, width, area, tilt angle, and formation time of the PEAs associated with $22 \mathrm{MC}$ and 29 non-MC events and we find no difference between the two populations. According to current ideas on the relation between flares and CMEs, the PEA is formed together with the CME flux-rope structure by magnetic reconnection. Our results indicate that at the Sun flux ropes form during CMEs in association with both $\mathrm{MC}$ and nonMC events; however, for non-MC events the flux-rope structure is not observed in the interplanetary space because of the geometry of the observation, i.e. the location of the spacecraft when the structure passes through it.
\end{abstract}

KEYWORDS: Flares; Coronal Mass Ejections; Post-eruption arcades; Flux rope; Magnetic cloud

\section{Introduction}

Two-ribbon flares are characterized by a pair of bright ribbons observed in $\mathrm{H} \alpha$ and ultraviolet (UV) images. The ribbons are located on either side of a magnetic polarity inversion line and they separate from each other as the flare progresses. Two-ribbon flares are often associated with filament eruptions and coronal mass ejections (CMEs). After the launch of the filament, long-lived arcades are formed connecting the two ribbons across the polarity inversion line. The emerged assembly of arches is called a post-eruption arcade (PEA). The PEAs are observed in multiple wavelengths and are known also as long duration (or decay) events (LDEs; Pallavicini, Serio, and Vaiana, 1977) in the X-ray observations. The erupting filament becomes the core of the associated CME (Webb and 
Hundhausen, 1987; Gopalswamy et al., 2003), thus PEAs are considered as surface signatures of CMEs (Tripathi, Bothmer, and Cremades, 2004).

The CSHKP magnetic reconnection model is widely accepted as a model of two-ribbon flares and CME onsets. The acronym "CSHKP" stands authors of this model, Carmichael (1964), Sturrock (1966), Hirayama (1974), Kopp and Pneuman (1976). This model suggests that during a filament eruption, a current sheet is formed and magnetic reconnection occurs below the erupting filament. The reconnected field lines arching downward to the solar surface form the PEA, while the upward reconnected field lines envelop the erupting filament and form the flux-rope structure (see e.g., Longcope and Magara 2004; Longcope and Beveridge, 2007). This model explains well the characteristics of two-ribbon flares: the separation of flare ribbons, the development of the PEAs, and the association with filament eruptions and CMEs (Bruzek 1964; Sheeley et al. 1975; Kahler 1977; Munro et al. 1979; Harra-Murnion et al. 1998; Yurchyshyn 2008). $\mathrm{H} \alpha$ flare ribbons form the feet of the PEAs at the chromospheric level. At the coronal level, the PEAs are observed as a collection of loops in X-rays, EUV, and even microwaves (e.g., Hanaoka et al., 1994; McAllister et al., 1996; Gopalswamy et al., 1999; 2003; Tripathi, Bothmer, and Cremades, 2004). The model is also supported by the relationship between the reconnected magnetic flux during flares and the magnetic flux of MCs observed near Earth, as reported by Qiu et al. (2007).

There is a general consensus that large PEAs appearing near the central meridian of the Sun are good indicators of geoeffective CMEs. The Bastille Day flare on 2000 July 14, with a bright EUV arcade (see Figure 1a, see also Aschwanden and Alexander 2001), caused an intense geomagnetic storm with a minimum Dst of -301 nT. During an earlier event on 1994 April 14, when coronographic observations of CMEs were more limited, the Soft X-ray Telescope on the Yohkoh satellite observed a giant PEA that extended $150^{\circ}$ in longitude. An Earth-directed CME was expected and an alert of a geomagnetic storm was issued (McAllister et al. 1996). We should note that the X-ray intensity of the event was very low; the X-ray Sensor on the Geostationary Operational Environmental Satellites (GOES) did not detect any significant X-ray enhancement. This case showed that a large-scale PEA is a good indicator of CMEs arriving at Earth and causing geomagnetic storms. The Extreme-ultraviolet Imaging Telescope (EIT) on 

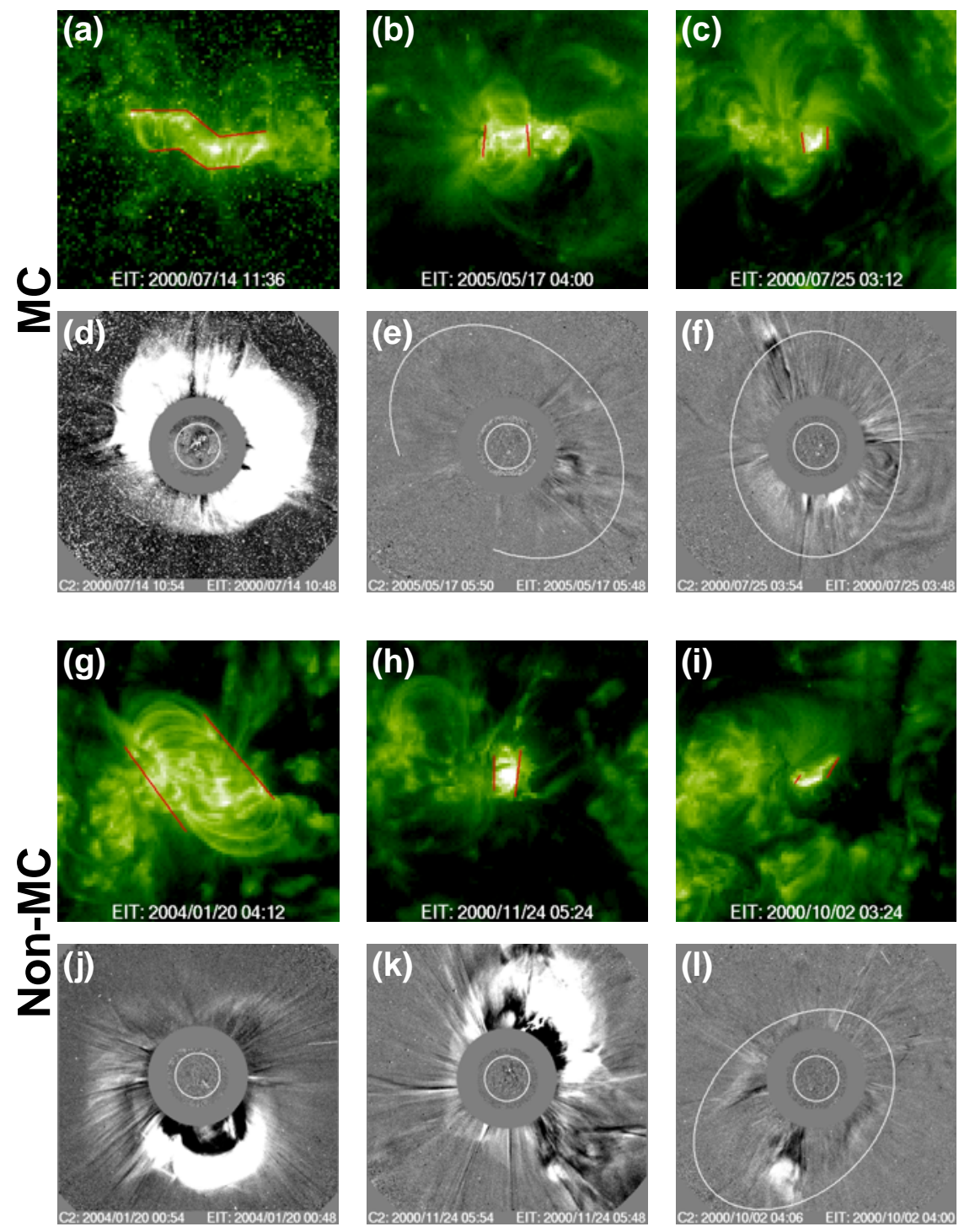

Figure 1 - PEAs observed by EIT (1st and 3rd rows) and the associated CMEs observed by LASCO (2nd and 4th rows). The top 2 rows show the solar sources of MC events, while the bottom 2 rows are for non-MC events.

board the Solar and Heliospheric Observatory (SOHO) mission started observing PEAs routinely since 1996 and helped to identify the solar sources of CMEs observed by the Large Angle and Spectrometric Coronagraph (LASCO; Brueckner et al., 1995).

Two Coordinated Data Analysis workshops (CDAWs) were held in 2010 and 2011 to address the question: Do all CMEs have a flux-rope structure? This question derives from the fact that only a fraction of the interplanetary CMEs 
(ICMEs; i.e. the plasma and magnetic field structure in interplanetary space corresponding to CMEs from the Sun) are observed as flux ropes or magnetic clouds at 1 AU (MCs; Burlaga et al. 1981). ICMEs without an observable fluxrope structure are called "ejecta" or non-MCs.

It has been suggested that non-MCs may have a flux-rope structure but that it may not be observed in single-point measurements at $1 \mathrm{AU}$ if the observing spacecraft passes through the periphery of the ICME (Gopalswamy, 2006). If this were the case, one does not expect any difference between PEAs associated with MCs and non-MCs at the Sun. Such a view has been supported by the fact that a flux rope can be fit to all white-light CMEs near the Sun, irrespective of their 1AU manifestation as MCs or non-MCs (Xie, Gopalswamy, and St. Cyr, 2013). As CMEs propagate away from the Sun, the trajectories of those CMEs associated with non-MCs become non radial (Xie et al., 2013; Kim et al., 2013) due to deflection by nearby coronal holes (Mäkelä et al., 2013). Other possibilities are CME-CME interactions leading to complex ejecta (Burlaga, Plunkett, and St. Cyr, 2002) or inherent non flux-rope structure at the Sun (Gosling, 1990). Gopalswamy et al. (2013) have shown that both MCs and non-MCs have charge-state enhancements at $1 \mathrm{AU}$, which is an indication of flare reconnection and flux-rope formation at the Sun, thus ruling out the non flux-rope structure suggested by Gosling (1990).

If CMEs resulting in non-MCs do not have a flux-rope structure and if the fluxrope structure of the $\mathrm{MC}$-associated $\mathrm{CMEs}$ is formed by magnetic reconnection, as suggested by the CSHKP model, we should be able to find differences between the MC-associated and the non-MC-associated PEAs. The aim of this paper is to examine this possibility by investigating the differences of the PEAs observed in EUV between the two populations.

\section{Data Set and Analysis}

The data set used in this study consists of the CDAW events selected from the list of shock-driving ICMEs listed in Gopalswamy et al. (2010). From the more than 200 events listed in Gopalswamy et al. (2010), 59 events originating from the disk center $\left( \pm 15^{\circ}\right.$ from the central meridian) were selected to form the CDAW list. If one assumes that all CMEs have a flux-rope structure then many of the IP drivers 
at $1 \mathrm{AU}$ should be MCs because solar eruptions launched from disk center are expected to hit Earth head-on. However, only 24 events were MCs and the remaining 35 events were classified as ejecta or non-MCs. Comparisons of these two populations were the suggested agenda of the workshops.

A careful analysis of the selected 59 events was carried out by the meeting participants during and after workshops. It turned out that the revised solar source of 5 events (No. 6, 11, 12, 22, and 55) did not meet the original location criteria (for details see Gopalswamy et al., 2013). Therefore, the number of events in the CDAW list was reduced to 54. In this study we have excluded two events (No. 1 and 58) because of EIT data gaps. We have also excluded one event (No. 40) because no flare brightening was identified after the filament eruption.

Table 1 summarizes the properties of the 51 PEAs analyzed in our study. Note that the event numbers (column 1) are not sequential because we use the event numbers of the original 59 events. The ICME type is given in the column 2: MC for magnetic clouds and EJ (ejecta) for non-MC. General information on the solar sources are given in columns $3-7$ (column 3: the flare date in yyyy/mm/dd format; column 4: the flare start time as hh:mm in UT; column 5: the flare location in the heliographic coordinates; column 6: the soft X-ray flare class; column 7: the CME speed in $\mathrm{km} \mathrm{s}^{-1}$ ). Columns 8 - 12 give the PEA information: the length in $\mathrm{Mm}$, the width in $\mathrm{Mm}$, the size in $\mathrm{Mm}$, the tilt angle in degree, and the PEA developing time in hours (see Section 3 for their definitions).

In 31 events, out of the 51 analyzed, we could see well-developed PEAs. Figure 1a shows a PEA observed during the so-called Bastille Day event on 2000 July 14 (No. 19). The X-ray class of the associated flare was X5.7 and the flare duration was 40 minutes. This event was associated with an Earth-directed CME which arrived at Earth 38 hours later as an MC. This event provides a good example of a bright, large PEA associated with an MC. Figure 1g shows another bright PEA but this one was associated with a non-MC (No. 47). The flare was an LDE flare (duration $>4$ hours) with peak X-ray class C5.5. The flare occurred at S13W09 and was associated with a halo CME (see Figure 1j). Two days after the corresponding ICME was observed by the Advanced Composition Explorer (ACE). The ICME was classified as non-MC since no clear rotation of the magnetic field was observed. 
In 10 events, we could identify long-lived non-arcade loops (see Figure 1b; No. 54) or short-lived arcades (see Figure 1h; No. 28). These events differ a little bit from the typical appearance of PEAs (long-lived arcades). In the remaining10 events, we could see short-lived non-arcade flaring loop. Figure 1c shows an example of an M8.0 flare at 02:43 UT on 2000 July 25 (No. 21). The flare was compact $(<45 \mathrm{Mm})$ and impulsive (11 min). Usually, such compact impulsive flares are likely to be confined (Yashiro et al., 2006), but this flare was associated with an EIT dimming and a faint halo CME (Figure 1f). The halo CME appeared in LASCO/C2 at 03:30 UT with an apparent speed of $528 \mathrm{~km} \mathrm{~s}^{-1}$ and, 3 days later, the associated ICME was observed as an MC. One could not necessarily classify these as PEAs but we have included them in the analysis because, according to the CSHKP flare-CME model, bright flare loops and PEAs are not physically distinct.

The solar source of the non-MC event on 2000 October 5 (No. 25) was a halo CME that appeared in the LASCO/C2 field of view at 03:50 UT on October 3 (Figure 11). The main body of the CME appeared in the south-east quadrant and the faint envelope surrounding it expanded to cover the occulting disk of the LASCO/C2 coronagraph. The associated flare was the C4.1 flare at S09E07 at 02:48 UT on the same day. The flare was impulsive, so the EIT image observed 27 min after the flare peak (Figure 1i) does not show an arcade. These two examples show that occasionally poor arcade signatures are observed both in MCs and non-MCs.

\section{Results}

In order to investigate the spatial properties of the PEAs, we measured their lengths, widths, and areas. Since PEAs grow larger in time, we determined their properties when the size reached its maximum. We visually determined the footpoints of the PEAs in EUV images and measured the length, which corresponds to the two flaring ribbons in $\mathrm{H} \alpha$ images. The red lines of Figure 1 show the locations of the footpoints of each PEA. We define the length of the PEAs as the average length of the two ribbons.

Figure 2a shows the distribution of the lengths of PEAs associated with MCs. The lengths are distributed widely from $29 \mathrm{Mm}$ to $313 \mathrm{Mm}$ with an average of $120 \mathrm{Mm}$. Figure $2 \mathrm{~b}$ shows the same as $2 \mathrm{a}$ but for non-MC events. Similarly, the 

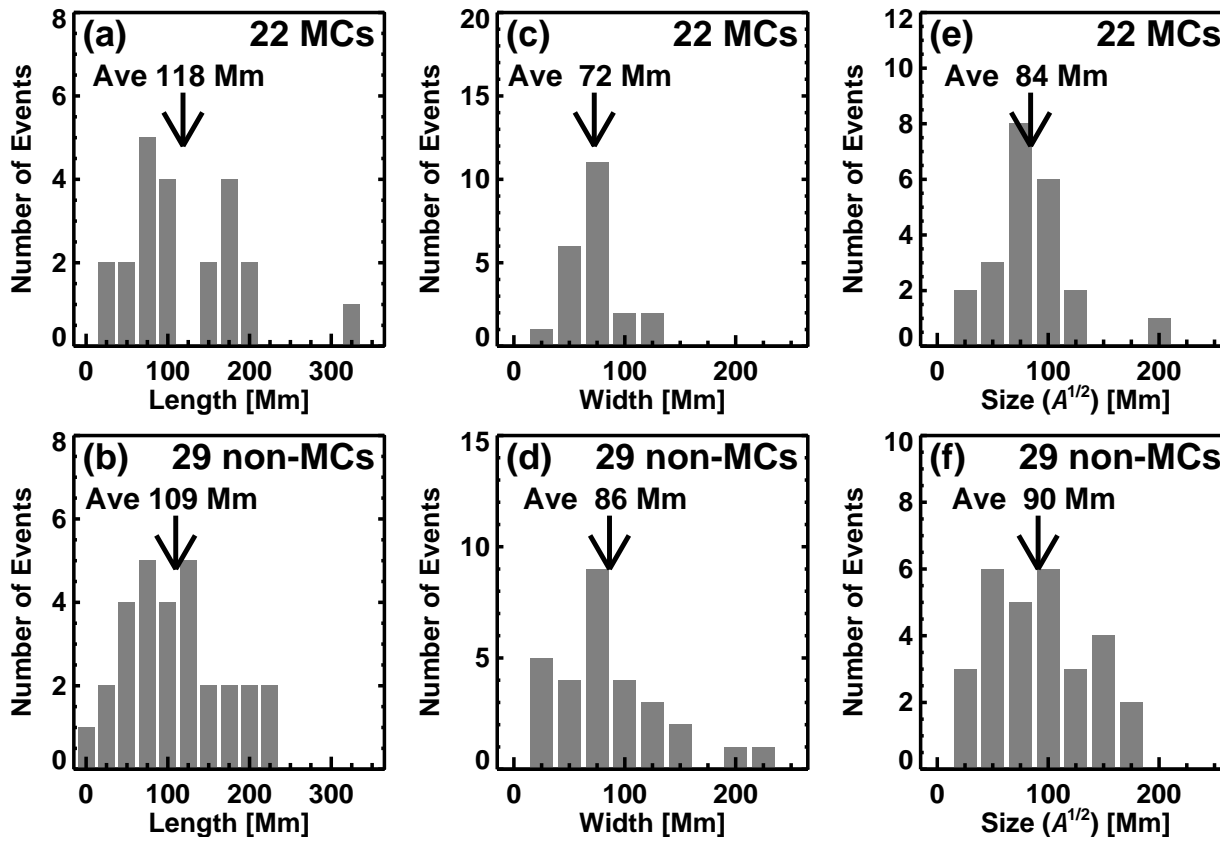

Figure 2 - Distributions of lengths (left), widths (center), and sizes $\left(A^{1 / 2}\right.$; right) for MC (top) and non-MC (bottom) events. The averages of each distribution are shown in the plot.

lengths of non-MC associated PEAs are distributed widely from $9 \mathrm{Mm}$ to 215 $\mathrm{Mm}$. The average length is slightly shorter but the difference in average length between the MC and non-MC events is not significant. Tripathi, Bothmer, and Cremades (2004) examined 236 PEAs using SOHO/EIT and reported that the heliographic length of the PEAs ranged from $2^{\circ}$ to $40^{\circ}$, which correspond to 24 $\mathrm{Mm}$ to $486 \mathrm{Mm}$. The lengths of PEAs associated with MCs are distributed within the range of Tripathi et al., but for the non-MCs cases they are not. The minimum length of $9 \mathrm{Mm}$ is out of the range because our data sets include compact flares without the arcade structure (see Figure 1i). We include them because flare loops and PEAs are not physically distinct according to the CSHKP model.

The middle panels compare the width distributions of the PEAs of MCassociated and non-MC-associated events. The width of each PEA is defined as an average distance between footpoints at the two ends. The average widths are similar in both populations. The width distribution of the MC-associated PEAs is narrower than that of the non-MC events.

The right-hand panels of Figure 2 compare the PEA size for the MC-events and non-MC events. We define the PEA sizes as square roots of the areas $\left(A^{1 / 2}\right)$ 

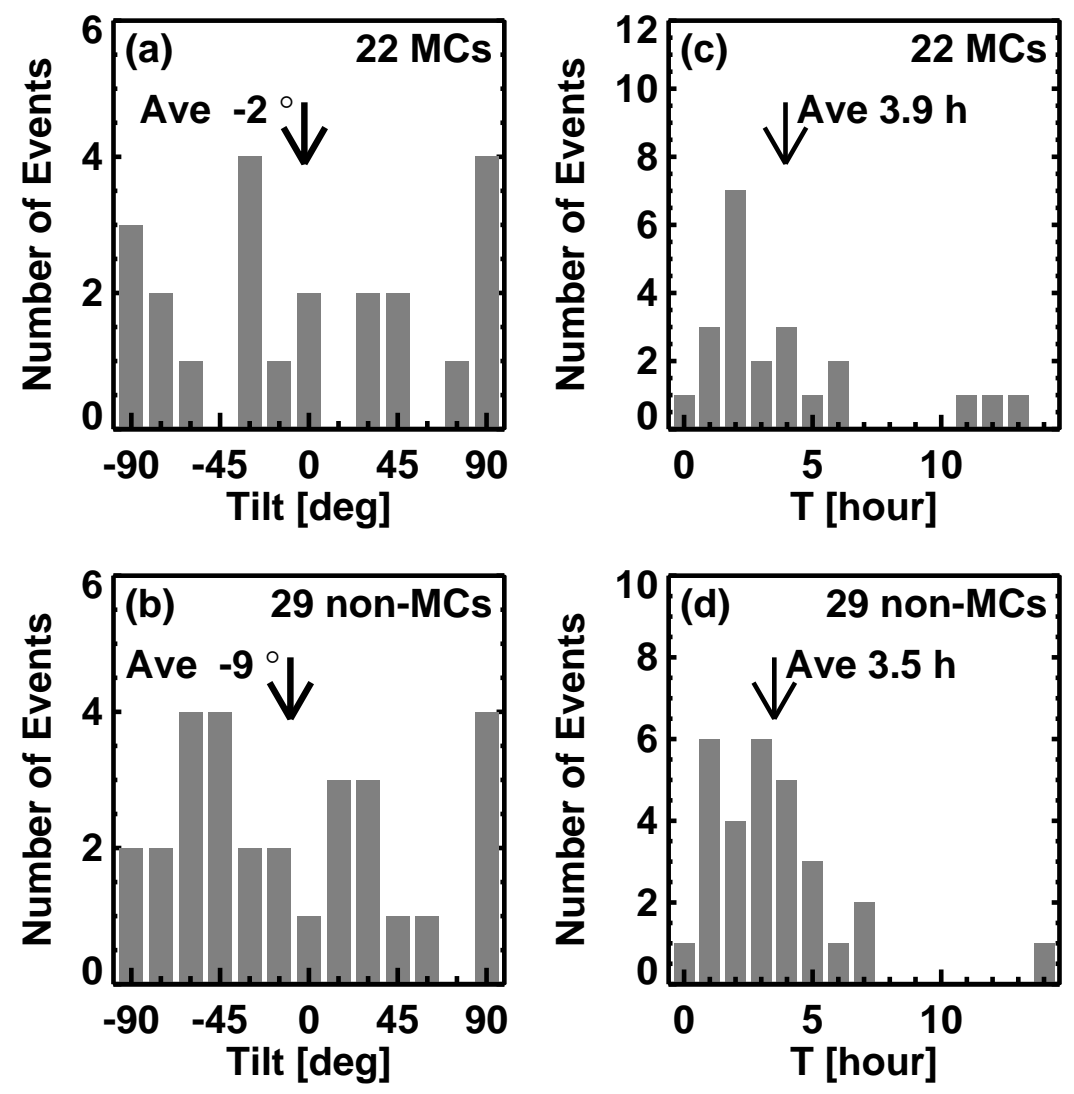

Figure 3 - Distributions of PEA tilt angles (left) and PEA developing times (right) for MC (top) and non-MC events (bottom).

between the two ribbons. We found that the PEA size distributions are very similar in both populations. The average PEA size is $85 \mathrm{Mm}$ for the $\mathrm{MC}$ events and $90 \mathrm{Mm}$ for the non-MC events.

We define the tilt angle of the PEAs as the angle between the PEA axis and the East-West line, measured counterclockwise in degrees. A horizontal PEA has a tilt angle within $\pm 10^{\circ}$. For example, the PEA of the Bastille Day event (Figure 1a) has a tilt angle of $-9^{\circ}$. A vertical PEA (e.g. Figures $1 \mathrm{~b}, 1 \mathrm{c}$, and $1 \mathrm{~h}$ ) a tilt angle larger than $80^{\circ}$ or lower than $-80^{\circ}$. With this definition, tilt angles $+90^{\circ}$ and $-90^{\circ}$ are identical. A PEA axis is determined as the straight line between the two mid points of the ribbons at its ends. We used the same definition even for curved PEAs. The direction of the flux-rope axis is not considered here because it is not a property of the PEA itself.

Figure 3a shows the distribution of the tilt angle of the MC-associated PEAs. The tilt angles are widely distributed from $-90^{\circ}$ to $90^{\circ}$. No clear trend is found in 
our sample. Figure $3 \mathrm{~b}$ is the same as Figure $3 \mathrm{a}$ but for non-MC events. We could not find any preferable tilt angle or any difference between the two distributions. One could expect more vertical PEAs than horizontal ones because ordinary sunspots are aligned horizontally, thus, the neutral line between them orients vertically. However, active regions producing many flares and CMEs are highly sheared and complex, thus, such simple consideration could not be valid.

In order to investigate the temporal properties of the PEAs, we use the time between the flare start and the PEA peak, defined as the time when a PEA is fully developed. We refer to this as the PEA developing time. PEA peak times are visually determined, so this is a somewhat subjective parameter. For the same event, different observers would select a different EIT frame as the PEA peak time. Our internal person-to-person check indicated that the errors between different observers are typically within 2 EIT frames or 24 minutes.

The right-hand panels of Figure 3 show the distribution of the PEA developing time for MC-associated (top) and non-MC-associated (bottom) events. As we saw in the other parameters, both distributions are very similar. The PEA developing time ranges from 24 minutes to 15 hours with an average of 3.9 hours for MCevents and 3.5 hours for non-MC-events, respectively. The average developing time is approximately half of the average lifetime (6.8 hours; Tripathi, Bothmer, and Cremades, 2004).

Since PEAs are the essential ingredients of solar flares, one might expect a relation between PEA and CME properties: the larger PEAs are more likely to be associated with faster CMEs. Since all our events originated the disk center, the observed speed represents the expansion speed which is correlated with the radial speed (Gopalswamy et al., 2009, 2012). Figure 4a plots the PEA size $\left(A^{1 / 2}\right)$ against the CME speed, but we cannot see any clear relation between them. The correlation coefficient is 0.08 for both $\mathrm{MC}$ and non-MC events and 0.09 for all data points.

It is known that the CME kinetic energy is proportional to the $\mathrm{X}$-ray peak intensity, but the correlation coefficient is only 0.54 (Hundhausen 1999, Yashiro and Gopalswamy 2009). Figure $4 \mathrm{~b}$ shows the correlation between the X-ray peak intensity and the CME speed. The correlation coefficient for all data points increases to 0.71 . 

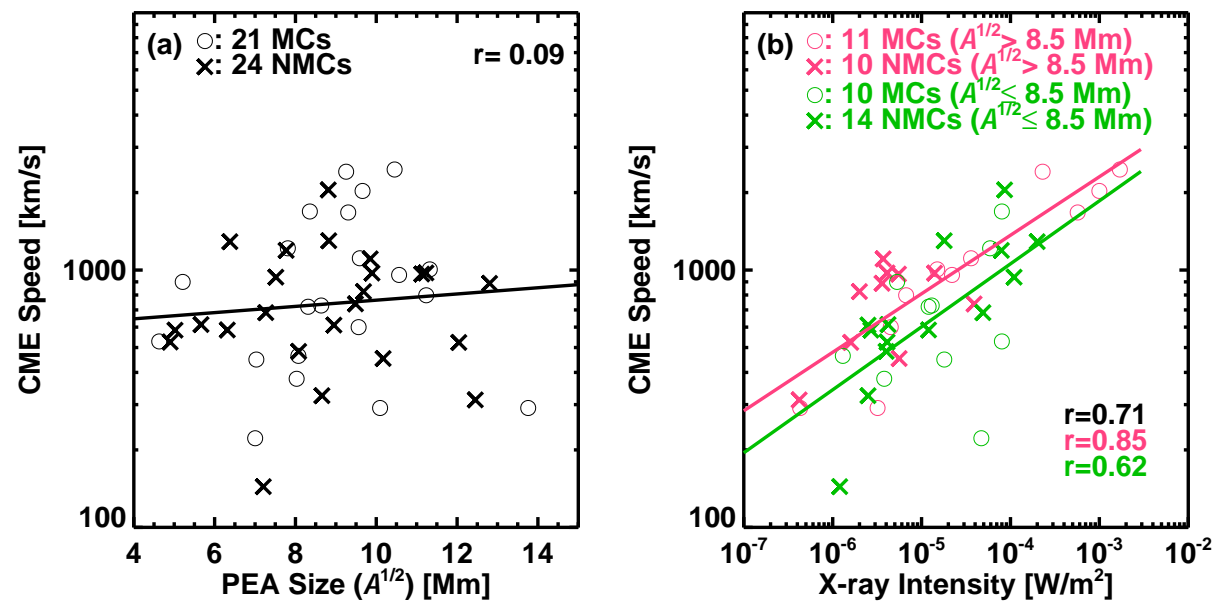

Figure $4-(a)$ Plot of the PEA sizes $\left(A^{1 / 2}\right)$ and the CME speeds. Circles and crosses denote the MC-associated and non-MC-associated events, respectively. (b) Plot of the peak X-ray intensity and the CME speed. Six events lacking an observed X-ray flare are excluded from the plot. Events with large $\left(A^{1 / 2}>8.5 \mathrm{Mm}\right)$ and small sizes $\left(A^{1 / 2} \leq\right.$ $8.5 \mathrm{Mm})$ are shown in pink and green, respectively.

We divided our sample into 4 groups based on the ICME structure, i.e. MC (circle) or non-MC (crosses) event, and on the PEA area, i.e. large $\left(A^{1 / 2}>8.5 \mathrm{Mm}\right)$ or small $\left(A^{1 / 2} \leq 8.5 \mathrm{Mm}\right)$ PEA. The circles and crosses are highly overlapped so we cannot see any difference between the MC and non-MC events. On the other hand we can see a difference between the large (pink) and small (green) PEAs. For a given flare intensity, the speeds of CMEs associated with larger PEAs are higher on average. The correlation coefficient is 0.85 for large and 0.62 for small PEA events.

\section{Summary and Conclusions}

Historic events, e.g. the Bastille Day Event on 2000 July 14 and the giant X-ray arcade formation on 1994 April 14, have convinced us that energetic CMEs are likely to be associated with a post-flare arcade formation. This idea is supported by the CSHKP model (e.g. Longcope and Magara 2004, Longcope and Beveridge 2007), i.e. the flux-rope structure of a CME is formed by magnetic reconnection which is also responsible for the formation of the PEA. If the non-MC associated 
CMEs do not have a flux-rope structure, we expect some difference in the PEA properties between $\mathrm{MC}$ and non-MC events. We find no difference in the PEA properties between the $\mathrm{MC}$ and non-MC populations.

In Figure 1 we show that the MC-associated CMEs are not necessarily associated with large PEAs. A CME associated with a compact impulsive flare was observed as an MC near Earth. Therefore, the association of a large PEA is not a necessary condition for a CME to have a flux-rope structure. In Figures 2 and 3 we compared the PEA properties between $\mathrm{MC}$ and non-MC events and found that there are no significant differences in the PEA properties between the two populations. In Figure 4 we found that the CME speeds weakly depend on the PEA sizes: the CMEs associated with larger PEAs tend to be faster than those associated with smaller ones. These results indicate that there is no difference in the properties of PEAs between the $\mathrm{MC}$ and non-MC events during the $\mathrm{CME}$ launch.

Xie, Gopalswamy, and St. Cyr (2013) applied a flux-rope model (Krall and St. Cyr, 2006) to the CMEs of the CDAW events and found that on average CMEs associated with MCs (non-MCs) are deflected towards (away from) the disk center. Kim et al. (2013) examined the CME direction parameter (Moon et al. 2005) and found that the parameter is smaller for non-MC events indicating that non-MC-associated CMEs are deflected away from the Sun-Earth line. Mäkelä et al. (2013) reported that different CME deflections between MC and non-MC events can be explained by the influence of coronal holes. Many studies suggest that there are significant differences in the CME launch direction between MC and non-MC events. Gopalswamy et al. (2013) found no clear difference in the flare properties of $\mathrm{MC}$ and non-MC events. In addition, we found that there is no significant difference in the properties of post-flare arcades. All these results are consistent with the view that at the Sun a CME flux-rope structure forms both in the $\mathrm{MC}$ and non-MC events, but the flux-rope structure is not observed in interplanetary space for non-MC events because of geometrical reasons.

\section{Acknowledgement}

We would like to thank the local organizers of the LWS CDAW meetings in San Diego, USA, and Alcalá de Henares, Spain. This research was supported by NASA grants NNX10AL50A and NNG11PL10A. SOHO is an international cooperation project between ESA and NASA. 


\section{Reference}

Aschwanden, M.J., Alexander, D.: 2001, Flare plasma cooling from $30 \mathrm{MK}$ down to $1 \mathrm{MK}$ modeled from Yohkoh, GOES, and TRACE observations during the Bastille Day event (14 July 2000). Solar Phys. 204, 91-120.

Brueckner, G.E., Howard, R.A., Koomen, M.J., Korendyke, C.M., Michels, D.J., Moses, J.D., Socker, D.G., Dere, K.P., Lamy, P.L., Llebaria, A., Bout, M.V., Schwenn, R., Simnett, G.M., Bedford, D.K., Eyles, C.J.: 1995, The Large Angle Spectroscopic Coronagraph (LASCO). Solar Phys. 162, 357-402.

Bruzek, A.: 1964, On the Association Between Loop Prominences and Flares. Astrophys. J. 140, 746.

Burlaga, L., Sittler, E., Mariani, F., Schwenn, R.: 1981, Magnetic loop behind an interplanetary shock - Voyager, Helios, and IMP 8 observations. J. Geophys. Res. 86, 6673-6684.

Burlaga, L. F., Plunkett, S.P., St. Cyr, O.C.: 2002, Successive CMEs and complex ejecta. J. Geophys. Res. 107, 1266.

Carmichael, H.: 1964, In: Hess, W.N. (ed.) Physics of Solar Flares, NASA SP-50, 451.

Gopalswamy, N.: 2006, Properties of interplanetary coronal mass ejections. Space Sci. Rev. 124, 145-168.

Gopalswamy, N., Dal Lago, A., Yashiro, S., Akiyama, S.: 2009, The expansion and radial speeds of coronal mass ejections. Cent. Eur. Astrophys. Bull. 33, 115.

Gopalswamy, N., Mäkelä, P., Akiyama, Xie, H., Yashiro, S., Reinard, A.A.: 2013, The Solar Connection of Enhanced Heavy Ion Charge States in the Interplanetary Medium: Implications for the Flux-Rope Structure of CMEs, Solar Phys. in this issue.

Gopalswamy, N., Mäkelä, P., Yashiro, S., Davila, J.M.: 2012, The Relationship Between the Expansion Speed and Radial Speed of CMEs Confirmed Using Quadrature Observations of the 2011 February 15 CME. Sun and Geosphere 7, 7.

Gopalswamy, N., Nitta, N., Manoharan, P.K., Raoult, A., Pick, M.: 1999, X-ray and radio manifestations of a solar eruptive event. Astron. Astrophys. 347, 684.

Gopalswamy, N., Shimojo, M., Lu, W., Yashiro, S., Shibasaki, K., Howard, R.A.: 2003, Prominence Eruptions and Coronal Mass Ejection: A Statistical Study Using Microwave Observations. Astrophys. J. 586, 562.

Gopalswamy, N., Xie, H., Mäkelä, P., Akiyama, S., Yashiro, S., Kaiser, M.L., Howard, R.A., Bougeret, J.-L.: 2010, Interplanetary shocks lacking type II radio bursts. Astrophys. J. 710, 1111.

Gosling, J.T.: 1990, Coronal Mass Ejections and Magnetic Flux Ropes in Interplanetary Space. Geophys. Monogr. Ser. 58, AGU, Washington, 343-364.

Hanaoka, Y., Kurokawa, H., Enome, S., Nakajima, H., Shibasaki, K., Nishio, M., et al.: 1994, Simultaneous observations of a prominence eruption followed by a coronal arcade formation in radio, soft X-rays, and Ha. Publ. Astron. Soc. Japan 46, 205-216.

Hirayama, T.: 1974, Theoretical model of flares and prominences. I: Evaporating Flare Model. Solar Phys. 34, 323-338. 
Hundhausen, A.: 1999, Coronal Mass Ejections. In: Strong, K.T., Saba, J.L.R., Haisch, B.M., Schmelz, J.T. (eds.) The Many Faces of the Sun: A Summary of the Results from NASA's Solar Maximum Mission, 143.

Harra-Murnion, L. K., Schmieder, B., van Driel-Gesztelyi, L., Sato, J., Plunkett, S. P., Rudawy, P., Rompolt, B., Akioka, M., Sakao, T., Ichimoto, K.: 1998, Multi-wavelength observations of POST flare loops in two long duration solar flares. Astron. Astrophys. 337, 911-920.

Kahler, S.: 1977, The morphological and statistical properties of solar X-ray events with long decay times. Astrophys. J. 214, 891-897.

Kim, R.-S., Gopalswamy, N., Cho, K.-S., Moon, Y.-J., Yashiro, S.: 2013, Propagation characteristics of CMEs associated magnetic clouds and ejecta. Solar Phys., submitted (this issue).

Kopp, R.A., Pneuman, G.W.: 1976, Magnetic reconnection in the corona and the loop prominence phenomenon, Solar Phys. 50, 85-98.

Krall, J., St. Cyr, O.C.: 2006, Flux-rope coronal mass ejection geometry and its relation to observed morphology. Astrophys. J. 652, 1740-1746.

Longcope, D.W., Beveridge, C.: 2007, A Quantitative, Topological Model of Reconnection and Flux Rope Formation in a Two-Ribbon Flare. Astrophys. J. 669, 621-635.

Longcope, D.W., Magara, T.: 2004, A Comparison of the Minimum Current Corona to a Magnetohydrodynamic Simulation of Quasi-Static Coronal Evolution. Astrophys. J. 608, 11061123.

Mäkelä, P., Gopalswamy, N., Xie, H., Mohamed, A., Akiyama, S., Yashiro, S.: 2013, Coronal Hole Influence on the Observed Structure of Interplanetary CMEs. Solar Phys., in this issue.

McAllister, A.H., Dryer, M., McIntosh, P., Singer, H., Weiss, L.: 1996, A large polar crown coronal mass ejection and a "problem" goemagnetic storm: April 14-23, 1994. J. Geophys. Res. 101, 13497-13515.

Moon, Y.J., Cho, K.S., Dryer, M., Kim, Y.H., Bong, S.C., Chae, J., Park, Y.D.: 2005, New Geoeffective Parameters of Very Fast Halo Coronal Mass Ejection. Astrophys. J. 624, 414-419.

Munro, R.H., Gosling, J.T., Hildner, E., MacQueen, R.M., Poland, A.I., Ross, C.L.: 1979, The association of coronal mass ejection transients with other forms of solar activity. Solar Phys. 61, 201-215.

Pallavicini, R., Serio, S., Vaiana, G.S.: 1977, A survey of soft X-ray limb flare images - The relation between their structure in the corona and other physical parameters. Astrophys. J. 216, 108-122.

Sheeley, N. R., Jr., Bohlin, J. D., Brueckner, G. E., Purcell, J. D., Scherrer, V. E., Tousey, R., Smith, J. B., Jr., Speich, D. M., Tandberg-Hanssen, E., Wilson, R. M.: 1975, Coronal changes associated with a disappearing filament. Solar Phys. 45, 377-392.

Sturrock, P.A.: 1966, Model of the High-Energy Phase of Solar Flares. Nature 211, 695-697.

Tripathi, D., Bothmer, V., Cremades, H.: 2004, The basic characteristics of EUV post-eruptive arcades and their role as tracers of coronal mass ejection source regions. Astron. Astrophys. 422, 337-349. 
Qiu, J., Hu, Q., Howard, T.A., Yurchyshyn, V.B.: 2007, On the magnetic flux budget in lowcorona magnetic reconnection and interplanetary coronal mass ejections. Astrophys. J. 659, 758-772.

Webb, D.F., Hundhausen, A.J.: 1987, Activity associated with the solar origin of coronal mass ejections. Solar Phys. 108, 383-401.

Xie, H., Gopalswamy, N., St. Cyr, O.C.: 2013, Near-Sun flux rope structure of CMEs. Solar Phys. in this issue.

Yashiro, S., Akiyama, S., Gopalswamy, N., Howard, R.A.: 2006, Different Power-Law Indices in the Frequency Distributions of Flares with and without Coronal Mass Ejections. Astrophys. $J$. Lett. 650, L143-L146.

Yashiro, S., Gopalswamy, N.: 2009, Statistical relationship between solar flares and coronal mass ejections. In: Gopalswamy, N., Webb, D.F. (eds.) IAU Symposium 257, Universal Heliophysical Processes, Cambridge Univ. Press, London, 233-243.

Yurchyshyn, V.: 2008, Relationship between EIT Posteruption Arcades, Coronal Mass Ejections, the Coronal Neutral Line, and Magnetic Clouds. Astrophys. J. Lett. 675, L49-L52. 
Table 1. List of the CDAW events and the post-eruption arcade parameters

\begin{tabular}{|c|c|c|c|c|c|c|c|c|c|c|c|}
\hline \multirow[b]{2}{*}{ No. } & \multirow[b]{2}{*}{$\begin{array}{l}\text { ICME } \\
\text { Type }^{\text {a }}\end{array}$} & \multicolumn{4}{|c|}{ Flare } & \multirow{2}{*}{$\begin{array}{c}\text { CME } \\
\text { Speed } \\
{[\mathrm{km} / \mathrm{s}]}\end{array}$} & \multicolumn{5}{|c|}{ Post-Eruption Arcade } \\
\hline & & Date & $\begin{array}{l}\text { Time } \\
\text { [UT] }\end{array}$ & Location & Class $^{b}$ & & $\begin{array}{l}\text { Length } \\
{[\mathrm{Mm}]}\end{array}$ & $\begin{array}{l}\text { Width } \\
{[\mathrm{Mm}]}\end{array}$ & $\begin{array}{l}\text { Size } \\
{[\mathrm{Mm}]}\end{array}$ & $\begin{array}{c}\varphi^{c} \\
\text { [deg] }\end{array}$ & $\begin{array}{c}T^{\mathrm{d}} \\
\text { [hour] }\end{array}$ \\
\hline 02 & $\mathrm{MC}$ & $1997 / 05 / 12$ & $04: 42$ & N21W08 & C1.3 & 464 & 77 & 67 & 65 & 83 & 12.1 \\
\hline 03 & EJ & $1997 / 12 / 06$ & $10: 00$ & N45W10 & EP & 397 & 210 & 189 & 169 & -45 & 13.9 \\
\hline 04 & EJ & $1998 / 05 / 01$ & $22: 36$ & S18W05 & M1.2 & 585 & 62 & 28 & 40 & -68 & 1.0 \\
\hline 05 & EJ & $1998 / 05 / 02$ & $13: 31$ & S15W15 & $\mathrm{X} 1.1$ & 938 & 74 & 42 & 56 & 6 & 1.4 \\
\hline 07 & EJ & $1998 / 11 / 04$ & $07: 13$ & N17W01 & C1.6 & 523 & 135 & 159 & 145 & -48 & 4.4 \\
\hline 08 & EJ & 1998/11/09 & $17: 03$ & N15W05 & C2.5 & 325 & 59 & 103 & 75 & -12 & 4.2 \\
\hline 09 & $\mathrm{MC}$ & $1999 / 04 / 13$ & $01: 45$ & N16E00 & B4.3 & 291 & 188 & 65 & 102 & -32 & 2.3 \\
\hline 10 & EJ & $1999 / 06 / 24$ & $12: 04$ & N29W13 & C4.1 & 975 & 169 & 82 & 126 & -37 & 3.7 \\
\hline 13 & EJ & $1999 / 09 / 20$ & $03: 58$ & S20W05 & EP & 604 & 215 & 90 & 153 & 28 & 3.8 \\
\hline 14 & EJ & 1999/10/17 & $23: 22$ & S30E15 & C1.2 & 144 & 50 & 50 & 52 & 89 & 2.4 \\
\hline 15 & EJ & $2000 / 01 / 18$ & $17: 07$ & S19E11 & M3.9 & 739 & 136 & 72 & 90 & 35 & 2.1 \\
\hline 16 & $\mathrm{MC}$ & $2000 / 02 / 17$ & $20: 17$ & S29E07 & M1.3 & 728 & 100 & 69 & 75 & -84 & 1.5 \\
\hline 17 & EJ & $2000 / 07 / 07$ & $08: 42$ & N17E10 & C5.6 & 453 & 90 & 119 & 103 & 89 & 5.1 \\
\hline 18 & EJ & $2000 / 07 / 08$ & $22: 58$ & N18W12 & C4.0 & 483 & 83 & 60 & 65 & -34 & 2.8 \\
\hline 19 & $\mathrm{MC}$ & $2000 / 07 / 14$ & $10: 03$ & N22W07 & $\mathrm{X} 5.7$ & 1674 & 159 & 59 & 87 & -9 & 1.6 \\
\hline 20 & EJ & $2000 / 07 / 23$ & 04:11 & S13W05 & $E P$ & 631 & 118 & 94 & 97 & 38 & 5.0 \\
\hline 21 & $M C$ & $2000 / 07 / 25$ & $02: 43$ & N06W08 & M8.0 & 528 & 29 & 45 & 21 & -86 & 0.5 \\
\hline 23 & $\mathrm{MC}$ & $2000 / 08 / 09$ & $15: 19$ & N20E12 & EP & 702 & 74 & 97 & 85 & -74 & 6.3 \\
\hline 24 & $M C$ & $2000 / 09 / 16$ & 04:06 & N14W07 & M5.9 & 1215 & 81 & 59 & 61 & -5 & 2.5 \\
\hline 25 & EJ & $2000 / 10 / 02$ & $02: 48$ & S09E07 & C4.1 & 525 & 9 & 70 & 24 & -90 & 0.6 \\
\hline 26 & MC & $2000 / 10 / 09$ & $23: 19$ & N01W14 & C6.7 & 798 & 165 & 85 & 126 & 27 & 2.1 \\
\hline 27 & MC & $2000 / 11 / 03$ & $18: 35$ & N02W02 & C3.2 & 291 & 313 & 106 & 189 & -34 & 10.6 \\
\hline 28 & EJ & $2000 / 11 / 24$ & $04: 55$ & N20W05 & $\mathrm{X} 2.0$ & 1289 & 53 & 30 & 41 & 87 & 0.5 \\
\hline 29 & EJ & $2001 / 02 / 28$ & $13: 22$ & S17W05 & B4.2 & 313 & 213 & 122 & 155 & 87 & 3.2 \\
\hline 30 & EJ & $2001 / 03 / 19$ & $04: 12$ & S20W00 & EP & 389 & 176 & 72 & 122 & 18 & 2.6 \\
\hline 31 & EJ & $2001 / 04 / 09$ & $15: 20$ & S21W04 & M7.9 & 1192 & 70 & 57 & 60 & -67 & 3.1 \\
\hline 32 & $\mathrm{MC}$ & $2001 / 04 / 10$ & 05:06 & S23W09 & $\mathrm{X} 2.3$ & 2411 & 146 & 65 & 86 & 83 & 2.1 \\
\hline 33 & $\mathrm{MC}$ & $2001 / 04 / 26$ & $11: 26$ & N20W05 & M1.5 & 1006 & 167 & 131 & 128 & -30 & 4.8 \\
\hline 34 & EJ & $2001 / 08 / 09$ & 08:00 & N11W14 & PEA & 479 & 97 & 233 & 140 & -80 & 7.2 \\
\hline 35 & EJ & $2001 / 10 / 09$ & $10: 46$ & S28E08 & M1.4 & 973 & 143 & 73 & 98 & 22 & 1.6 \\
\hline 36 & $\mathrm{MC}$ & $2002 / 03 / 15$ & $22: 09$ & S08W03 & M2.2 & 957 & 169 & 80 & 112 & 48 & 6.3 \\
\hline 37 & $\mathrm{MC}$ & $2002 / 04 / 15$ & 03:05 & S15W01 & M1.2 & 720 & 83 & 64 & 69 & 38 & 3.5 \\
\hline 38 & EJ & $2002 / 05 / 08$ & $12: 58$ & S12W07 & C4.2 & 614 & 34 & 31 & 32 & -66 & 1.4 \\
\hline 39 & $\mathrm{MC}$ & $2002 / 05 / 16$ & 00:11 & S23E15 & C4.5 & 600 & 100 & 114 & 91 & 72 & 3.2 \\
\hline 41 & EJ & $2002 / 05 / 27$ & $12: 36$ & N22E15 & C3.7 & 1106 & 129 & 79 & 97 & -62 & 3.0 \\
\hline 42 & EJ & $2002 / 07 / 15$ & $21: 03$ & N19W01 & M1.8 & 1300 & 93 & 66 & 78 & 58 & 4.5 \\
\hline 43 & $\mathrm{MC}$ & $2002 / 07 / 29$ & $10: 27$ & S10W10 & M4.7 & 222 & 91 & 29 & 49 & 32 & 0.6 \\
\hline 44 & $M C$ & $2003 / 08 / 14$ & $17: 12$ & S10E02 & C3.8 & 378 & 57 & 76 & 64 & -87 & 3.6 \\
\hline 45 & $\mathrm{MC}$ & $2003 / 10 / 28$ & $11: 00$ & S16E08 & $\mathrm{X} 17.2$ & 2459 & 200 & 66 & 109 & -37 & 2.4 \\
\hline 46 & $M C$ & $2003 / 10 / 29$ & $20: 37$ & S15W02 & $\mathrm{X} 10.0$ & 2029 & 101 & 86 & 93 & -77 & 4.2 \\
\hline 47 & EJ & $2004 / 01 / 19$ & $23: 46$ & S13W09 & C5.5 & 965 & 139 & 114 & 124 & -52 & 4.4 \\
\hline
\end{tabular}




\begin{tabular}{|r|c|r|c|c|c|r|r|r|r|r|r|}
\hline 48 & MC & $2004 / 07 / 22$ & $07: 41$ & N04E10 & C5.3 & 899 & 19 & 42 & 27 & 85 & 0.7 \\
\hline 49 & MC & $2004 / 11 / 06$ & $01: 40$ & N09E05 & M3.6 & 1111 & 163 & 52 & 92 & 2 & 1.7 \\
\hline 50 & EJ & $2004 / 12 / 08$ & $19: 34$ & N05W03 & C2.5 & 611 & 75 & 97 & 80 & -52 & 2.0 \\
\hline 51 & EJ & $2005 / 01 / 15$ & $05: 54$ & N16E04 & M8.6 & 2049 & 92 & 73 & 78 & 31 & 5.7 \\
\hline 52 & EJ & $2005 / 02 / 13$ & $10: 28$ & S11E09 & C2.7 & 584 & 30 & 27 & 25 & -89 & 0.5 \\
\hline 53 & MC & $2005 / 05 / 13$ & $16: 13$ & N12E11 & M8.0 & 1689 & 86 & 82 & 70 & -55 & 12.7 \\
\hline 54 & MC & $2005 / 05 / 17$ & $02: 31$ & S15W00 & M1.8 & 449 & 40 & 57 & 49 & 90 & 1.5 \\
\hline 56 & EJ & $2005 / 07 / 07$ & $16: 07$ & N09E03 & M4.9 & 683 & 84 & 36 & 53 & -60 & 0.9 \\
\hline 57 & EJ & $2005 / 08 / 31$ & $10: 26$ & N13W13 & C2.0 & 825 & 137 & 80 & 94 & -9 & 3.4 \\
\hline 59 & EJ & $2006 / 08 / 16$ & $14: 37$ & S16W08 & C3.6 & 888 & 209 & 155 & 164 & 10 & 7.2 \\
\hline
\end{tabular}

${ }^{\mathrm{a}} \mathrm{MC}=$ Magnetic cloud; $\mathrm{EJ}=$ Ejecta or non-MC

${ }^{\mathrm{b}} \mathrm{EP}=$ Eruptive prominence

${ }^{\mathrm{c}} \varphi=$ Tilt angle measured counterclockwise from East-West line in degree

${ }^{\mathrm{d}} T=$ PEA developing time. 